\title{
Intensité et spatialisation de l'îlot de chaleur urbain parisien à partir de données participatives
}

\author{
Malika Madelin ${ }^{1 *}$ et Vincent Dupuis ${ }^{2}$ \\ ${ }^{1}$ UMR 8586 PRODIG, Université de Paris, Paris, France \\ ${ }^{2}$ UMR 8234 PHENIX, Sorbonne Université, Paris, France
}

\begin{abstract}
Résumé - L'îlot de chaleur urbain est une des principales manifestations du climat urbain qui se caractérise par des températures plus élevées en ville qu'à la campagne et peut avoir des conséquences importantes pour les citadins en particulier lors des vagues de chaleur. Par convention, l'intensité de l'îlot est calculée, à partir des données des stations météorologiques de référence, comme la différence de température de l'air entre les zones urbaines et rurales environnantes. Dans cet article, nous cherchons à quantifier cette intensité et son extension sur la région parisienne, à partir d'un jeu de données massives participatives, que nous avons collectées depuis l'été 2017. Nous détaillons l'analyse critique de ces données non homogènes, absolument nécessaire avant leur exploitation scientifique. Nous montrons alors l'existence d'un îlot de chaleur urbain nocturne, dont l'intensité et l'extension spatiale varient selon les saisons et les conditions atmosphériques, avec une intensification lors des nuits radiatives (ciel clair, vent calme). In fine, nous spatialisons cet îlot pour des conditions radiatives et le comparons avec l'îlot de surface, produit à partir des températures de surface MODIS.
\end{abstract}

Mots-clés : climat urbain / îlot de chaleur urbain / données participatives / agglomération parisienne.

\begin{abstract}
Intensity and spatialisation of Paris urban heat island from crowdsourced data. The urban heat island is one the main characteristics of the urban climate that corresponds to higher temperatures in city centres compared to the countryside, a situation that can have important consequences on the dwellers' health. In this article, we aim at quantifying its intensity and its spatial extent over the Paris area from a massive crowdsourced dataset collected since summer 2017. We will detail the critical analysis done on this non homogeneous dataset as it is absolutely necessary prior to any scientific use. We will then show the existence of an urban heat island whose intensity and extent vary according to seasons and atmospheric conditions. In the end, we will compare theses new results with results from a study of the urban heat island based on surface temperature: we observe the same general features globally but the crowdsourced data show a much better temporal resolution and this provides new opportunities to study the dynamics of the urban heat island.
\end{abstract}

Keywords: urban climate / urban heat island / crowdsourced data / Paris area.

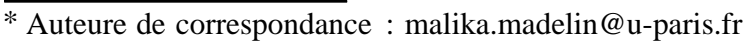

\section{Introduction}

L'îlot de chaleur urbain est une des principales manifestations du climat urbain (Oke, 1982 ; Stewart, 2011). Il se manifeste par des températures de l'air nocturnes généralement plus élevées dans le centre des villes que dans les campagnes environnantes. En effet, l'hétérogénéité des propriétés thermiques et des perméabilités des surfaces urbaines et rurales environnantes entraîne des flux radiatifs et convectifs différents, et donc des températures différentes des surfaces et de l'air sus-jacent. Un écart de plusieurs degrés entre les températures de l'air en ville et les températures dans la campagne environnante est alors très souvent observé, surtout lors des nuits de ciel clair et de vent calme (conditions radiatives). Ce phénomène peut amplifier les situations d'inconfort, en ville, lors des vagues de chaleur, voire le risque sanitaire pour les personnes les plus 
vulnérables (Alonso et Renard, 2020 ; Laaidi et al., 2012 ; Pascal et al., 2018 ; Sakhy, 2016).

L'intensité de l'îlot de chaleur urbain est traditionnellement estimée comme une différence des températures de l'air mesurées par des stations de référence dites urbaines d'une part et des stations dites rurales d'autre part (Oke, 1987; Stewart, 2011 ; Cantat, 2014; Foissard, 2015). Cette approche est simple mais reste limitée par le faible nombre de points et leur représentativité des contextes urbains et ruraux (Bigot et al., 2017). Pour affiner, des réseaux denses, à une échelle locale, se développent à l'initiative de groupes de recherche et/ou de collectivités territoriales (Alcoforado et Andrade, 2006; Masson et al., 2008 ; Muller et al., 2013 ; Skarbit et al., 2017 ; Richard et al., 2018; Foissard et al., 2019). D'autres approches s'appuient sur des mesures mobiles, principalement réalisées lors de conditions radiatives (e.g. les travaux de thèse de Foissard, 2015 et Le Bras, 2015), sur la modélisation (Wouters et al., 2013 ; Kastendeuch et Najjar, 2015 ; Le Bras, 2015) ou encore sur l'utilisation de données satellitaires (Dousset et al., 2011 ; Madelin et al., 2017 ; Le Roy et al., 2019).

Depuis quelques années, avec la diminution drastique du coût des composants électroniques, plusieurs entreprises (Ambient Weather, Bloomsky, Bresser, NetAtmo...) proposent des stations météo personnelles connectées, souvent associées à des plateformes de visualisation (site web, application mobile). Ce marché répond à un vif intérêt du grand public pour la météorologie, comme en témoigne le classement mensuel des applications les plus téléchargées : La Chaîne Météo sur mobile fait partie chaque mois des 10 premières applications et souvent dans les 5 premières en été (site web de L'Alliance pour les Chiffres de la Presse et des Médias, consultation en mars 2020). Si la production de données par des amateurs n'est pas nouvelle, ces nouvelles données participatives, «crowdsourcées », produites par le grand public, commencent à devenir massives et suscitent l'intérêt de la communauté scientifique, qui y voit une opportunité pour la recherche (Muller et al., 2015). À ce jour, il existe plusieurs réseaux, dont la répartition spatiale et la densité varient selon l'origine de l'entreprise, sa date de création ou encore le prix de l'objet connecté : par exemple, les réseaux privés Weather Underground (États-Unis) et Netatmo (France), ou encore la plateforme WOW Weather Observation Website lancée par MetOffice (Royaume-Uni) et déployée par plusieurs autres services météo nationaux (Australie, Belgique, Irlande, Nouvelle-Zélande, Pays-Bas, Suède), qui permet aux particuliers de partager leurs mesures faites à partir de stations compatibles. Ces réseaux complètent les réseaux nationaux souvent insuffisamment denses et les réseaux denses, à une échelle locale, déployés par des groupes de recherche et/ou des collectivités territoriales.

Ces données participatives ont donné lieu à plusieurs travaux récents que ce soit en météorologie (Mandement et Caumont, 2019), pour améliorer les systèmes de prévision à Moscou en complément de WRF - Weather Research and Forecasting (Uteuov et al., 2019) ou encore de données satellitaires et lidar à Oslo (Venter et al., 2020). Sur l'îlot de chaleur urbain, Chapman et al. (2017) sur Londres ont montré le lien avec les conditions de stabilité de l'atmosphère. Sur Berlin, Fenner et al. (2019) ont étudié l'intensité de l'îlot lors des vagues de chaleur. D'autres travaux (Fenner et al., 2017 sur Berlin ; Feichtinger et al., 2020 sur Vienne) ont analysé le lien avec les LCZ Local Climate Zones de Stewart et Oke (2012).

Une question centrale préalable à l'utilisation scientifique de ces nouvelles données est leur qualité. La critique des données, certes toujours essentielle, est alors ici encore plus cruciale : mesures faites par des stations ne possédant souvent pas d'abri radiatif, disposées à des hauteurs variables et dans des contextes environnementaux différents. À ce sujet, F. Meier et ses collaborateurs (2015 et 2017) sur les données Netatmo à Berlin furent les premiers à utiliser ces données et à développer une procédure systématique de tri des données de température provenant du réseau Netatmo. D'autres travaux, depuis, ont été réalisés pour qualifier les données de précipitations (de Vos et al., 2019) ou encore affiner la critique des données avec des critères spatiaux (Nipen et al., 2019).

Dans cet article, nous proposons une étude de 
l'îlot de chaleur parisien, de son intensité et étendue spatiale, à partir d'un jeu de données originales issu du réseau Netatmo, depuis l'été 2017. Le choix de la zone d'étude repose sur la disponibilité et surtout la densité des mesures des stations Netatmo autour de Paris. En outre, cette agglomération représente un espace à forts enjeux, avec une population importante de 12 millions d'habitants en Île-deFrance (recensement INSEE 2015), avec une densité de population en moyenne de $1000 \mathrm{hab} / \mathrm{km}^{2}$ et jusqu'à plus de $20000 \mathrm{hab} / \mathrm{km}^{2}$ pour la ville de Paris. Dans cet article, nous insistons en premier lieu sur la méthodologie de la critique des données, en discutant des similitudes ou non avec les approches citées précédemment. Puis, nous utilisons ce jeu de données, qualifié et original, pour étudier l'intensité de l'îlot de chaleur urbain à Paris, son évolution temporelle sur la période d'étude, ainsi que sa spatialisation pour les nuits de ciel clair et de vent calme.

\section{Un jeu de données météorologiques massives à critiquer}

\subsection{Collecte d'un jeu de données Netatmo autour de Paris}

Depuis l'été 2017, nous avons constitué une base de données des stations Netatmo autour de Paris, à partir de requêtes via une API (application programming interface), toutes les heures (dev.netatmo.com/apidocumentation/). Nous avons retenu ici les températures de l'air, mesurées par plus de 18000 stations avec un identifiant différent, dans un rayon de $70 \mathrm{~km}$ autour de Paris, ce qui représente un réseau particulièrement dense (figure 1). Chaque station est localisée par un couple de coordonnées longitude/latitude. Au total, nous avons plus de 20700 localisations géographiques (avec une marge de $50 \mathrm{~m}$ ) et identifiants uniques, chiffre plus élevé que le nombre d'identifiants de stations. En effet, un même identifiant dans la base avant pré-traitements peut avoir eu plusieurs localisations (déménagement, prêt, etc.).

Ce volume conséquent de stations sur l'agglomération parisienne, et donc de données (par exemple, près de 56 millions de lignes pour l'année 2019), peut s'expliquer par l'origine géographique de la startup Netatmo. Ces stations, achetées par des particuliers $(\sim 170 €)$, sont installées dans des contextes très différents, bien éloignés des normes de l'Organisation Mondiale de la Météorologie : en plein soleil, dans un jardin, à l'intérieur d'un appartement, sur un balcon au 18e étage, dans un abri météo, etc. Précisons que nous n'avons aucune métadonnée associée aux stations et que les informations de localisation géographique des stations sont déclaratives (connexion par wifi) et parfois automatiques (par le GPS d'un smartphone lors de l'installation).

En admettant des erreurs de localisation, la Figure 1 montre très clairement une surreprésentation des installations des stations dans les zones urbanisées, surtout dans Paris (tissu urbain continu), puis dans la proche couronne, en particulier dans les zones à revenus élevés (par exemple, les Hauts-de-Seine à l'ouest), à l'inverse de la Seine Saint-Denis au nord. Les stations étant installées par des particuliers, les zones éloignées des habitations, comme les forêts ou les territoires agricoles, présentent très logiquement une faible densité de capteurs météorologiques. Pour illustrer quantitativement, les territoires urbanisés qui représentent près de $26 \%$ de la superficie de la zone d'étude (figure 1) rassemblent plus de $90 \%$ des stations (24\% dans le tissu urbain continu et $67 \%$ dans le discontinu), alors que les zones agricoles (40\% de la zone) n'ont que $2 \%$ des stations. Notons que cette sur-représentation des contextes urbains est l'inverse des réseaux de référence, où au contraire il est difficile de mesurer les climats urbains (Bigot et al., 2017 ; Dahech et al., 2020 ; Rome et al., 2020).

\subsection{Sélection d'un jeu de données à partir d'une solide critique des données}

Avant toute exploitation scientifique des températures de l'air, nous avons réalisé une solide analyse critique de ces données non homogènes, absolument nécessaire étant donné les premières visualisations et comparaisons avec les données de référence. La méthodologie, décomposée en plusieurs étapes, repose d'abord et principalement sur la sélection des stations (figure 2), puis sur l'exclusion des données aberrantes. 


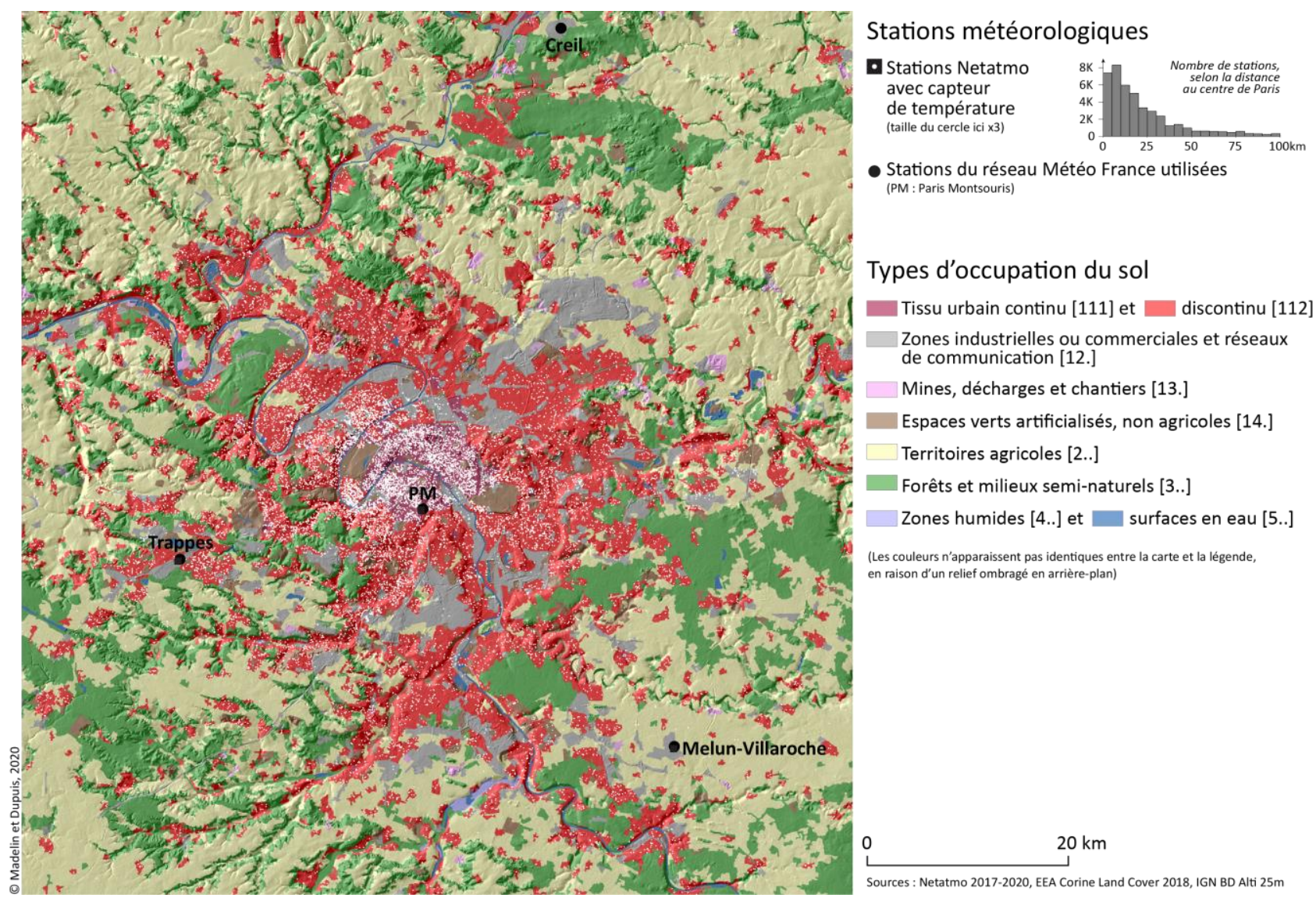

Figure 1. Le réseau des stations Netatmo autour de Paris, mesurant la température sur la période 2017-2020, et les principales occupations du sol. The Netatmo weather station network around Paris, recording temperatures during 2017-2020 period, and main land cover types.

\section{- Critère de localisation}

Dans un premier temps, nous avons étudié l'identifiant et la localisation des stations (figure 2, critère 1) : même si ces informations sont constantes sur la période juin 2017 à mars 2020 pour la très grande majorité des stations Netatmo, nous avons constaté des changements de coordonnées géographiques et, à l'inverse, des localisations identiques pour plusieurs identifiants. Dans le premier cas, il peut s'agir d'un déménagement, d'un changement de propriétaire, mais aussi de l'utilisation d'un smartphone (plusieurs points proches liés à une mauvaise réception, notamment à l'intérieur d'un bâtiment). Nous avons choisi de créer un nouvel identifiant pour les stations, prenant en compte ces modifications de localisation (pour des distances supérieures à $50 \mathrm{~m}$, sinon utilisation des coordonnées moyennes). Dans le second cas, nous avons éliminé les groupes de stations ayant des coordonnées identiques - au moins trois fois - et des identifiants différents (le maximum étant 658 fois pour le centre de Paris !). En d'autres termes, nous choisissons de considérer qu'il est peu probable d'avoir plus de deux capteurs exactement au même endroit.

Ce critère de localisation est similaire à celui utilisé par Meier et al. (2017), même s'il laisse ici la possibilité d'un déménagement éventuel, d'un changement de propriétaire, etc. Cette étape a éliminé $13 \%$ des stations, mais en a rajouté quasiment autant avec la création du nouvel identifiant.

\section{- Critère de complétude temporelle}

La deuxième étape de la critique (figure 2 , critère 2) a consisté à sélectionner des stations selon un critère de complétude des données. Nous avons d'abord choisi les capteurs avec au moins un mois de données journalières, elles-mêmes calculées sur au moins 18 heures (ce qui peut s'apparenter aux $80 \%$ des données horaires exigés par la méthode de Meier et al. (2017). 
Figure 2. Méthodologie utilisée pour la critique de données des stations Netatmo. Methodology used for the quality check of the Netatmo station data.
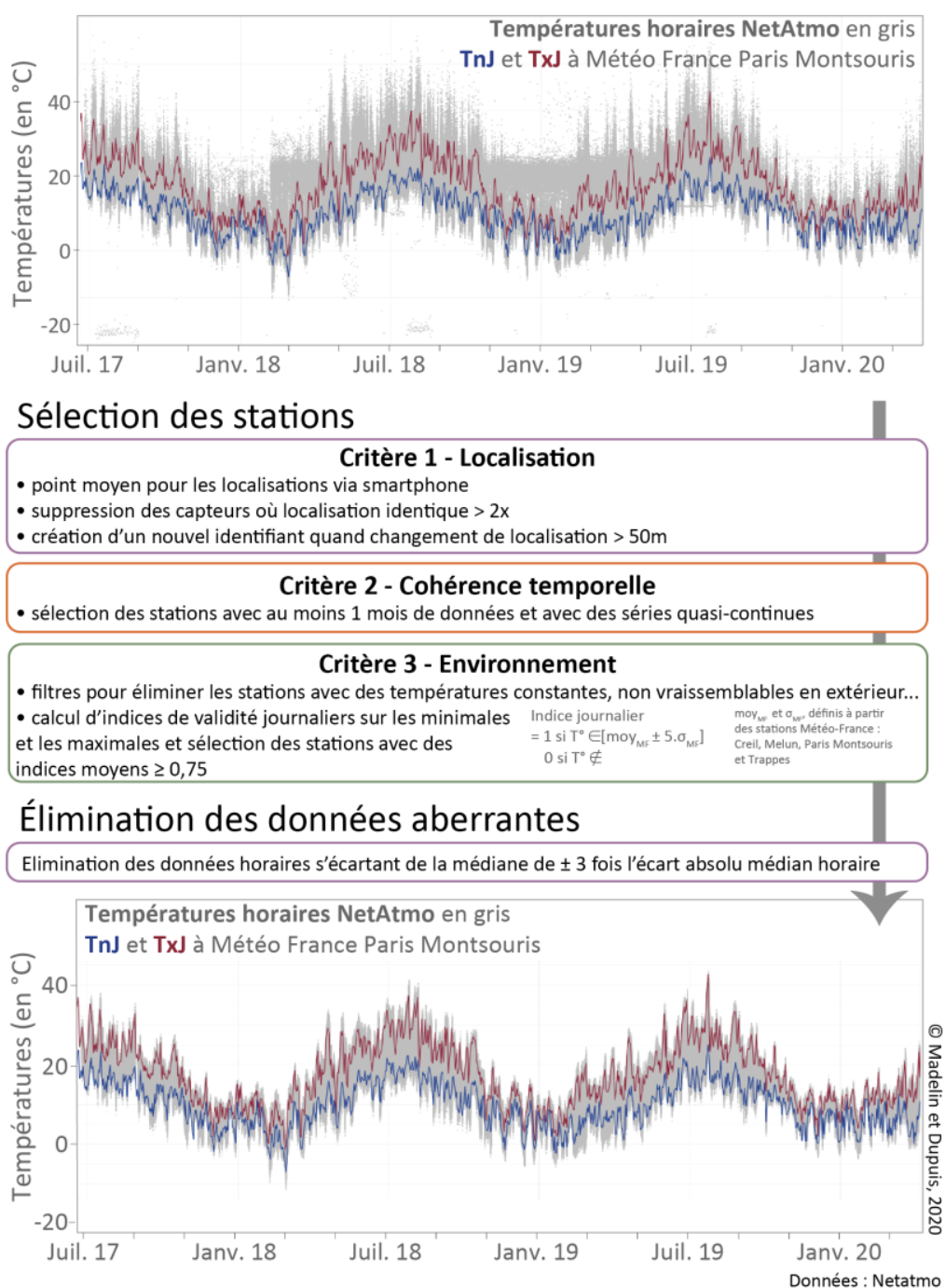

Ce critère, très discriminant, élimine plus de la moitié des stations, ce qui peut s'expliquer partiellement par la création de notre nouvel identifiant (étape précédente) et sans doute, aussi, par une utilisation très ponctuelle de ces objets connectés. Pour renforcer la sélection de séries complètes, cette fois-ci à l'échelle horaire, nous avons retenu les stations pour lesquelles $90 \%$ des données avaient un écart temporel entre deux données successives inférieur ou égal à $1 \mathrm{~h}, 95 \%$ inférieur ou égal à $2 \mathrm{~h}$ et $99 \%$ inférieur ou égal à $30 \mathrm{~h}$ (seuils choisis après examen des distributions statistiques des écarts et de l'observation des données brutes). Au terme de cette étape, nous retenons presque $40 \%$ des stations et près des $2 / 3$ des données initiales.

\section{- Critère d'environnement}

Puis, nous avons cherché à sélectionner les stations avec des données vraisemblables pour une température de l'air à l'extérieur et cohérentes avec les données de stations de référence, en l'occurrence celles de Météo France. En effet, nous avions observé des comportements particuliers pour des stations, caractéristiques a priori d'environnements différents: par exemple, des températures minimales proches de celles de Météo France et des températures maximales beaucoup plus élevées pouvaient laisser supposer une station au soleil. Des premiers filtres ont été appliqués : par exemple, élimination des stations avec de faibles écarts thermiques annuels ou encore avec une moyenne des températures minimales en hiver trop élevée. Puis nous avons calculé un indice de validité, par station, pour les températures minimales et maximales à partir de 4 stations de référence de Météo France (figure 1): Paris-Montsouris, Trappes (au WSW et à $27 \mathrm{~km}$ du centre de Paris), 
Melun-Villaroche (SSE, $37 \mathrm{~km}$ ) et Creil (N, $46 \mathrm{~km}$ ). Cet indice représente le pourcentage de données journalières comprises entre la moyenne journalière des quatre stations de référence \pm 5 écarts-types (seuil permettant d'éliminer les stations avec données aberrantes, tout en cherchant à conserver les contrastes climatiques locaux). Nous avons choisi de retenir les stations avec des indices de validité supérieurs à $75 \%$ pour les températures minimales et pour les maximales (figure 2, critère 3), soit $30 \%$ des stations initiales. Du fait de la nécessité d'avoir des données de stations de référence, cette étape est souvent négligée au profit d'une élimination statistique des données aberrantes (étape suivante).

\section{- Le jeu de données après élimination des données aberrantes}

Enfin, une fois les stations sélectionnées, nous avons appliqué une dernière vérification pour écarter les données parfois aberrantes des stations retenues (par exemple, une station mise quelques heures/jours à l'intérieur). En utilisant la méthode de Hampel (Hampel et al., 1986), pour chaque heure, les données s'écartant de la médiane pour l'ensemble des stations de \pm 3 fois l'écart absolu médian ont été éliminées.

Au final, la base de données comprend 6180 stations (figure 2, avant/après en bas), soit $53 \%$ du jeu initial en termes de températures horaires et un tiers des stations initiales, avec toutefois une forte hétérogénéité spatio-temporelle. Cette critique des données, longue et approfondie, est plus que nécessaire dans le cas de données participatives, où nous n'avons pas d'informations sur les choix initiaux (identification et localisation des stations, résolution temporelle, etc.) et ici aucune métadonnée.

\section{Intensité de l'îlot de chaleur urbain sur l'agglomération parisienne à partir des données Netatmo}

L'îlot de chaleur urbain est traditionnellement exprimé par la différence de température de l'air entre deux stations, l'une située dans la ville, l'autre dans la campagne avoisinante. Pour la région parisienne, O. Cantat (2004) avait ainsi utilisé les données des stations de Montsouris et de Melun (cf. figure 1) pour quantifier l'îlot. Pour s'approcher de cet indicateur usuel, de ce delta ville-campagne, nous avons sélectionné d'une part des stations dites « rurales », dans des contextes ruraux (dans les « territoires agricoles » et à plus de $30 \mathrm{~km}$ du centre de Paris) et d'autre part des stations localisées dans un contexte urbain (« tissu urbain dense » de la base Corine Land Cover, c'est-à-dire grosso modo Paris intramuros). Afin d'éviter des jeux de données trop dissymétriques (initialement 139 stations rurales $v s$. 929 stations urbaines), nous avons sélectionné une centaine de stations urbaines, les plus complètes. L'écart thermique est calculé s'il y a au moins 30 stations par heure et dans chaque groupe.

La Figure 3 représente la différence entre les médianes des températures horaires des stations urbaines et celles des rurales retenues, en fonction du temps (en abscisse les jours et en ordonnée les heures), par année. Pour quelques heures, nous n'avions pas assez de données pour calculer une différence robuste (ici en gris). Des variations peuvent s'observer d'un jour à l'autre, correspondant à différentes conditions atmosphériques. L'écart thermique est quasiment toujours positif, globalement plus faible lors des journées estivales (fort échauffement des zones agricoles, effet d'ombrage en ville) et, à l'inverse, plus fort la nuit, parfois intense pour certaines dates avec des conditions radiatives (ciel dégagé et vent calme). Par exemple, la deuxième quinzaine de février 2019, avec des conditions anticycloniques de blocage, sur une période exceptionnellement longue, ressort très nettement, avec des îlots de chaleur urbains nocturnes intenses : les nuits $(21 \mathrm{~h}$ 6 h UTC) du 12 au 28 février 2019 ont connu des différences d'au moins $3^{\circ} \mathrm{C}$ entre les médianes horaires des stations urbaines et celles des rurales et jusqu'à $7,5^{\circ} \mathrm{C}$ (médiane : $5,85^{\circ} \mathrm{C}$ ).

Pour résumer l'information contenue dans ce jeu de données, nous nous sommes intéressés à l'influence de la saisonnalité sur l'intensité de l'îlot de chaleur urbain. La Figure 4A montre la variation moyenne journalière, à l'échelle horaire, de l'écart présenté sur la Figure 3, selon les mois d'hiver (DJF), de printemps (MAM), d'été (JJA) et d'automne (SON). 

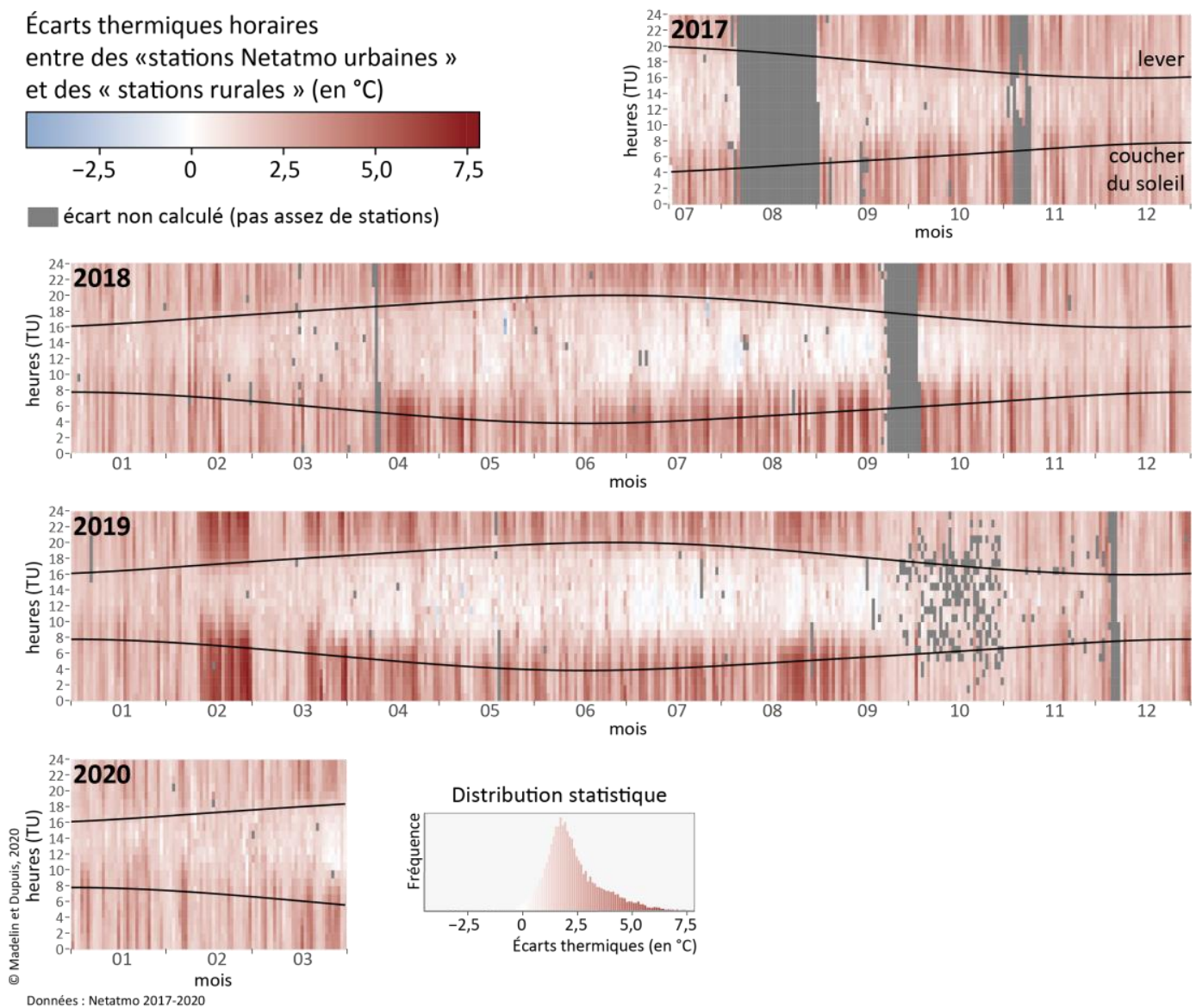

Figure 3. Écarts thermiques entre des stations Netatmo « urbaines » et « rurales », en fonction des heures et des jours et distribution statistique de ces écarts. 139 stations ayant une localisation dans un territoire agricole, selon la nomenclature Corine Land Cover 2018 et situées à plus de $30 \mathrm{~km}$ du centre de Paris sont qualifiées de « rurales »; les stations « urbaines » correspondent à une sélection d'environ 140 stations dans Paris intra-muros. Les écarts ne sont pas calculés si les effectifs de chaque groupe sont inférieurs à 30 stations. Temperature differences between Netatmo urban and rural stations as a function of the date and time of the studied period and statistical distribution. 139 stations located in rural areas according to the 2018 Corinne Land Cover classification and at a distance larger than $30 \mathrm{~km}$ from Paris center are considered as "rural". The urban stations are a subset of 140 stations located inside Paris city. The temperature differences are calculated only if at least 30 stations are available in each group.

Nous observons à nouveau que l'îlot de chaleur urbain est un phénomène nocturne (l'écart est plus fort la nuit qu'en journée), ainsi qu'un effet de saisonnalité : l'îlot est plus intense l'été (avec un écart moyen de plus de $3^{\circ} \mathrm{C}$ ) que l'hiver (écart moyen de $2,5^{\circ} \mathrm{C}$ ). Ce résultat est en accord avec le delta Paris-Montsouris - Melun (données de Météo France ; figure 4B) : un îlot de chaleur nocturne maximum en été $\left(2,4^{\circ} \mathrm{C}\right)$ et minimum en hiver $\left(1,3^{\circ} \mathrm{C}\right)$. La différence entre « $1^{\prime} \mathrm{ICU}$ Netatmo » et «l'ICU Météo France» est probablement attribuable à un biais de représentativité, les stations Netatmo étant plus fortement soumises à l'influence urbaine.

Enfin, pour résumer davantage et ne retenir qu'un chiffre, nous avons calculé la moyenne de l'intensité de l'îlot de chaleur nocturne à partir des stations Netatmo qui correspond ici à $2,9^{\circ} \mathrm{C}$ (médiane : $2,6^{\circ} \mathrm{C}$ ) ; à titre de comparaison, l'écart moyen entre les stations de référence Paris Montsouris et Melun est de $2^{\circ} \mathrm{C}$ (médiane : $1,8^{\circ} \mathrm{C}$ ) sur la même période. Il est important de préciser que l'écart moyen pour les données Netatmo est calculé à partir des écarts journaliers entre les médianes des minima des températures horaires des stations (entre $18 \mathrm{~h}$ la veille et $18 \mathrm{~h}$ le jour) des groupes « urbain » et « rural », alors que l'écart de référence est calculé à partir des températures minimales journalières pour Paris-Montsouris et Melun. 

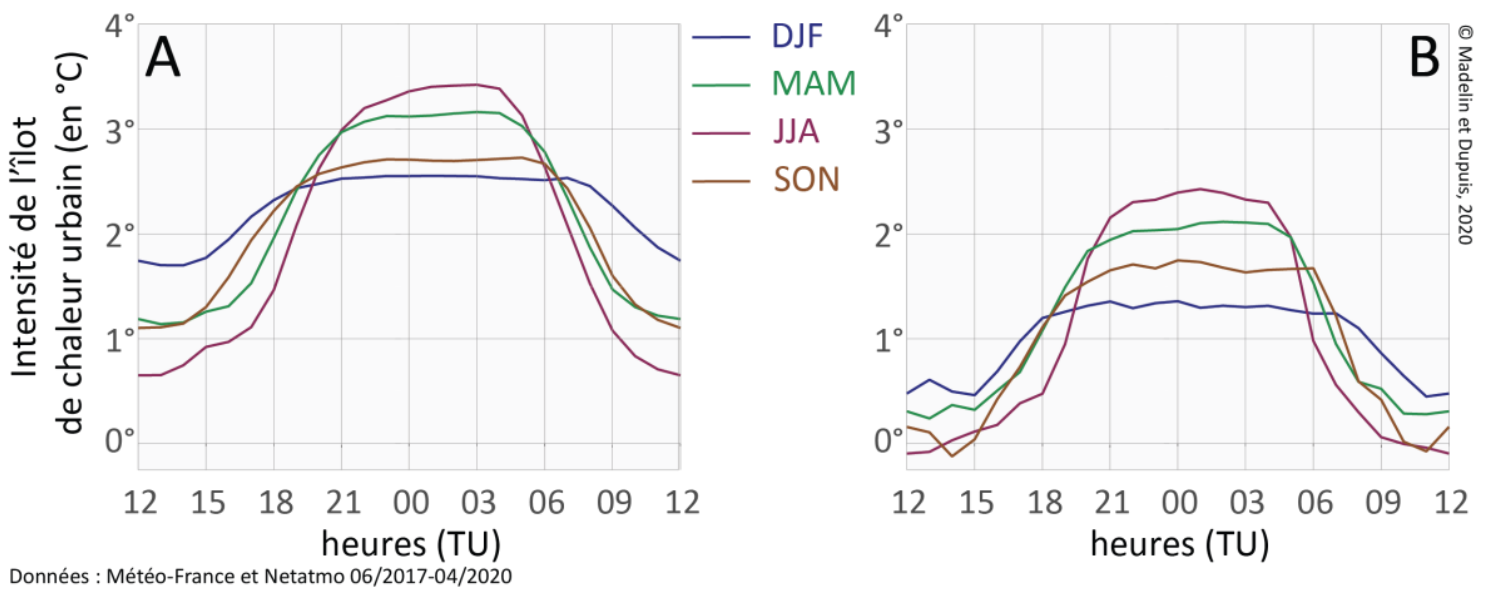

Figure 4. (A) Écart thermique moyen entre des stations Netatmo « urbaines » et « rurales », en fonction des heures et selon les mois d'hiver (DJF, décembre, janvier, février), de printemps (MAM, mars, avril, mai), d'été (JJA, juin, juillet, août) et d'automne (SON, septembre, octobre, novembre). (B) Écart thermique entre les stations Météo France de Paris-Montsouris et de Melun, moyenné de la même manière. (A) Temperature difference between Netatmo urban and rural stations averaged on the winter (DJF December, January, February), spring (MAM March, April, May), summer (JJA June, July, August) and autumn (SON September, October, November) months as a function of the time of the day. (B) Temperature difference between the Météo France Paris-Montsouris and Melun stations, averaged and plotted the same way.

Cet îlot nocturne moyen plus intense pour Netatmo, au-delà des différences de mesures entre les deux types de réseau, pourrait là encore témoigner d'une meilleure prise en compte des caractéristiques urbaines, mal restituées par la station de Montsouris en raison de sa situation dans un parc urbain.

\section{Spatialisation de l'îlot de chaleur urbain pour des nuits de ciel clair et de vent calme}

Au-delà de l'intensité de l'îlot de chaleur urbain, calculée classiquement comme le delta thermique entre urbain et rural, et pour appréhender la dimension spatiale du phénomène, nous nous sommes intéressés au champ de température et à son gradient.

La Figure 5 présente les variations de l'ensemble des températures mesurées par les stations Netatmo en fonction de la distance au centre de Paris pour deux dates, le 8 mai 2018 et le 13 septembre 2017, avec des conditions atmosphériques très différentes. Lors de la première nuit $(8 / 5 / 18)$, les conditions de ciel clair (marais barométrique) ont favorisé l'apparition d'un îlot de chaleur urbain intense, avec une décroissance marquée des températures jusqu'à environ $20 \mathrm{~km}$ autour de Paris (soit un gradient d'environ $5^{\circ} \mathrm{C}$ sur $20 \mathrm{~km}$ soit $\left.0,25^{\circ} \mathrm{C} / \mathrm{km}\right)$. À l'inverse, le 13 septembre 2017 était caractérisé par des conditions nuageuses, avec un front pluvieux : aucune structure spatiale n'est identifiable, les températures sont relativement homogènes sur l'ensemble de la zone. Pour ces deux dates, aucune structure spatiale n'est clairement visible en journée et les conditions atmosphériques expliquent la dispersion des températures observées.

Ces deux exemples montrent la particularité des conditions de ciel clair et de vent calme, propices à l'apparition d'un îlot de chaleur urbain nocturne intense. Nous avons cherché à spatialiser les températures sur l'ensemble de la zone d'étude pour ces conditions atmosphériques. Ce choix nous permet de plus de comparer les champs produits avec des données MODIS de températures de surface (Madelin et al., 2017 ; Madelin et Dupuis, 2018), c'est-à-dire de comparer les champs nocturnes de températures de l'air et de surface. Nous avons sélectionné les données Netatmo autour de $2 \mathrm{~h}$ UTC (qui correspond à peu près à l'heure de passage du satellite MODIS AQUA), pour les dates avec un ciel clair (à partir de la disponibilité des images MODIS) et un vent calme (moyenne des vitesses de vent moyen à Melun, entre $21 \mathrm{~h}$ et $6 \mathrm{~h}$ UTC, inférieure à $2 \mathrm{~m} / \mathrm{s}$ ) : au total, 34 dates, avec, pour chacune, au moins 1970 capteurs Netatmo. 

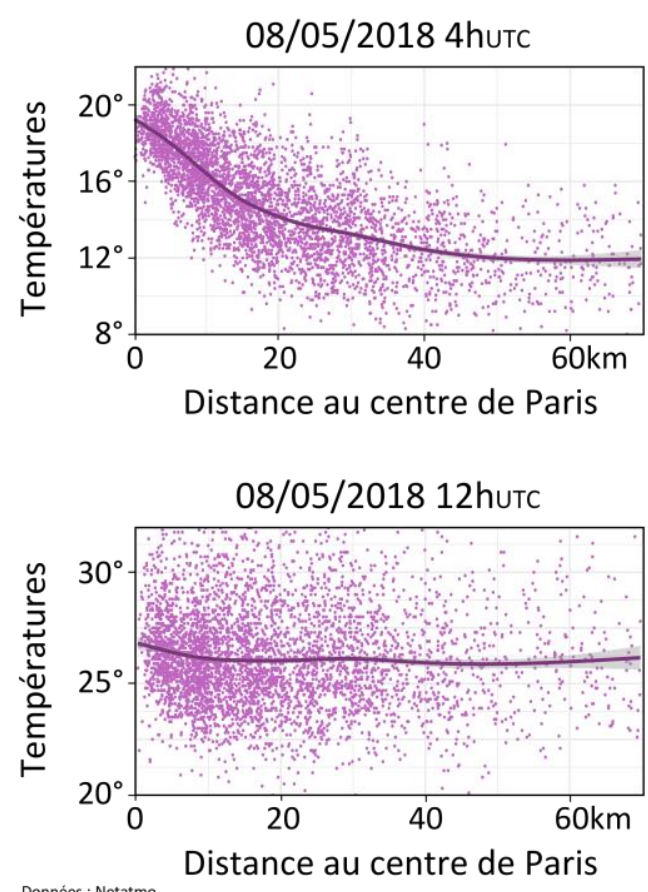
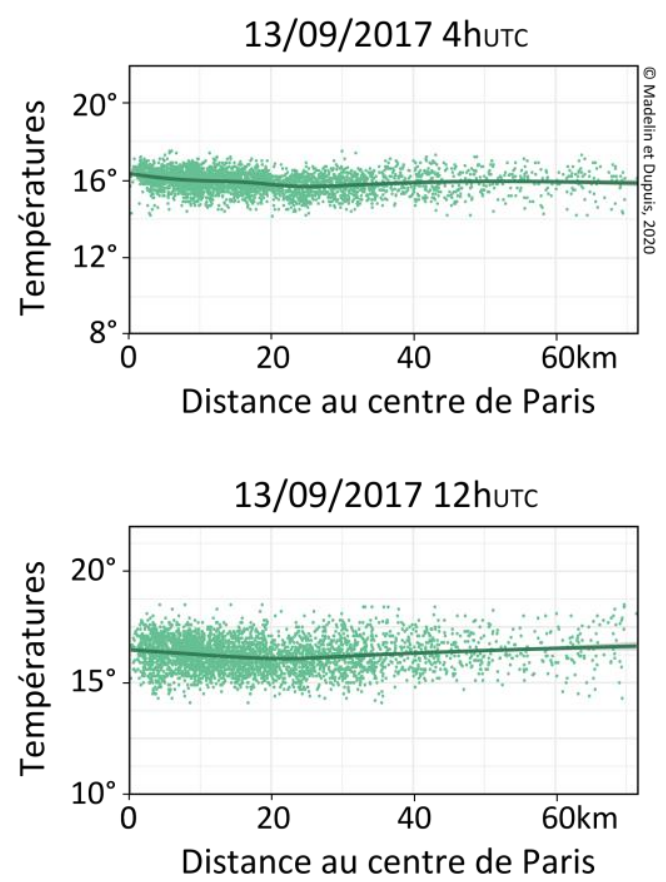

Figure 4. Deux exemples contrastés de la variation des températures selon la distance au centre de Paris. Two contrasting examples of temperature spatial distribution, around Paris.

Dans cette sélection, 11 dates sont au printemps (MAM), 10 en été (JJA), 7 en automne (SON) et 6 en hiver (DJF). Pour chacune des dates retenues, nous avons effectué une analyse variographique puis une interpolation des données de Netamo par krigeage ordinaire, sur une grille avec la même résolution que les images MODIS ( 1 km).

La Figure 6 représente les deux images des températures nocturnes, de l'air et de surface, lors de nuits de ciel clair et de vent calme, à 2 h UTC : à gauche (A), la médiane des interpolations des températures de l'air (Netatmo) et à droite (B), la médiane des températures de surface (MODIS). Les deux se basent sur les anomalies. Si des différences de répartition spatiale sont visibles, la forme et l'extension des deux îlots sont assez proches. Paris et sa proche couronne ressortent très nettement, avec des anomalies positives, et une légère extension selon 1'orientation nord-ouest/sud-est, dans les deux cas. Nous observons un décalage vers des plus faibles valeurs pour l'image médiane Netatmo par rapport à celle de MODIS, qui s'explique par une dominance des stations Netatmo au centre de l'agglomération (poids statistique important dans le calcul des anomalies, avant krigeages). Ces résultats sont globalement cohérents avec la littérature académique sur l'îlot à Paris. L'avantage des nouvelles données participatives est qu'il est possible de spatialiser l'îlot de chaleur urbain avec une résolution temporelle fine (horaire, voire subhoraire), et d'accéder ainsi à sa dynamique.

\section{Conclusion}

Dans cet article, nous avons cherché à montrer les potentialités qu'offraient les données participatives pour les études climatiques en nous intéressant en particulier aux données de températures issues du réseau de stations météo personnelles Netatmo et à l'îlot de chaleur urbain parisien. Ces données massives, disponibles, mais complexes du fait du manque d'information sur le contexte des mesures nous ont conduits à réaliser une analyse critique très sélective des données, basée sur des critères de localisation, de complétude temporelle et d'environnement (près de $50 \%$ du jeu de données initial est écarté).

Cette question de la critique des données participatives constitue à la fois un préalable à leur utilisation et un sujet de recherche en soi comme le montrent les nombreux travaux cités en introduction (dont Meier et al., 2017). 


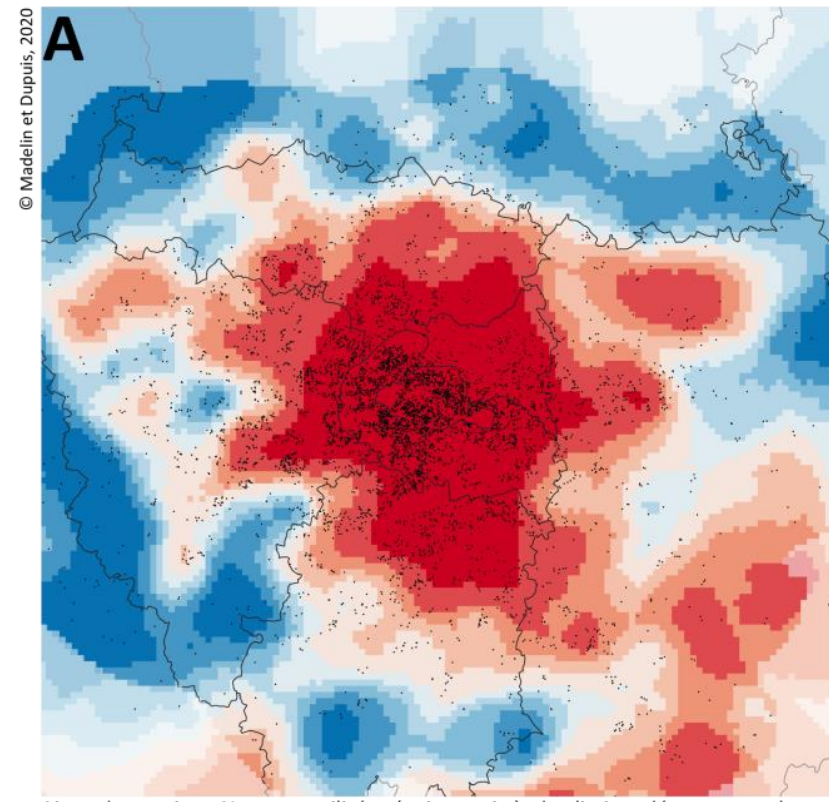

Ajout des stations Netatmo utilisées (points noirs), des limites départementales et d'un masque blanc pour une erreur standard $>1,5^{\circ} \mathrm{C}$.

Médiane des interpolations réalisées à partir des anomalies des températures de l'air des stations Netatmo (en ${ }^{\circ} \mathrm{C}$ ), 2 hUTC, pour 34 dates* de ciel clair et de vent calme

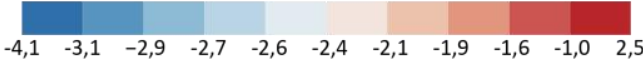

Discrétisations selon les déciles

* 11 dates pour MAM, 10 pour JJA, 7 pour SON et 6 pour DJF

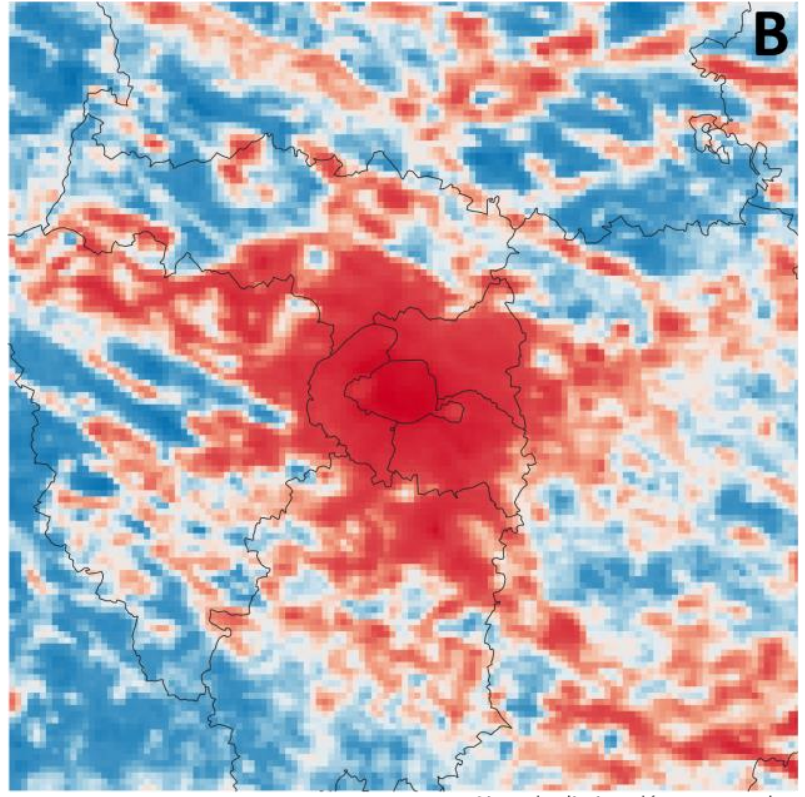

$20 \mathrm{~km}$

Ajout des limites départementales.

Médiane des anomalies des températures de surface de MODIS AQUA (en $\left.{ }^{\circ} \mathrm{C}\right)$, 2 2 hUTC, pour 34 dates* de ciel clair et de vent calme

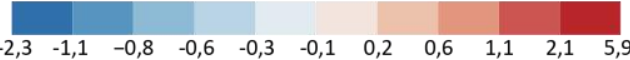

Données : Netatmo 2017-2020, MODIS AQUA 2017-2020

Figure 6. Images médianes $(A)$ des interpolations des anomalies de températures de l'air Netatmo et (B) des anomalies de températures de surface MODIS, pour des nuits de ciel clair et de vent calme. Median images (A) of spatial interpolations of air temperature anomalies from Netatmo stations and $(B)$ of surface temperature anomalies from MODIS, during nights with clear sky and calm wind.

Ainsi, nous aurions pu aller plus loin et ajouter des critères d'homogénéité spatiale en écartant les comportements trop différents entre stations proches (Venter et al., 2020). Néanmoins, après la critique développée dans cet article, nous avons pu étudier l'îlot de chaleur urbain parisien sur la période 2017-2020 à partir des données des stations Netatmo sélectionnées. Nous avons montré que son intensité varie selon les saisons et les conditions atmosphériques et que quantitativement les données Netatmo sont en accord avec les données du réseau de référence Météo France tout en apportant une résolution spatiale bien supérieure. En pratique, les deux réseaux sont complémentaires, le réseau Netatmo étant principalement urbain alors que les stations Météo France sont principalement installées dans des zones périurbaines et rurales (Kounkou-Arnaud et Brion, 2018). Enfin, nous avons cherché à comparer l'îlot de chaleur urbain à l'îlot de chaleur urbain de surface (à partir des données satellitaires MODIS) lors des situations radiatives, dans la poursuite de précédents travaux (Madelin et al., 2017 ; Madelin et Dupuis, 2018). Les résultats sont en accord avec l'étude réalisée récemment par B. Le Roy et al. (2019) sur la période 2000-2016, qui ont comparé les données Météo France interpolées aux données MODIS. Enfin, même si les données participatives utilisées ici nécessitent une analyse critique conséquente (qui pourra ultérieurement être affinée) et donc même s'il faut être plutôt prudents, nous aimerions insister sur les potentialités et les opportunités qu'elles offrent, par leur résolution spatio-temporelle, et il serait intéressant d'approfondir la dynamique d'apparition et disparition de l'îlot de chaleur en couplant ces données avec des simulations numériques.

Remerciements : Les auteurs souhaitent remercier la mise à disposition des environnements serveurs de développement et de publication de $\mathrm{R}$ par la TGIR Huma Num. 


\section{Références}

Alcoforado M., Andrade H., 2006. Nocturnal urban heat island in Lisbon (Portugal): main features and modelling attempts. Theoretical and Applied Climatology, 84, 151-159.

Alonso L., Renard F., 2020. A Comparative Study of the Physiological and Socio-Economic Vulnerabilities to Heat Waves of the Population of the Metropolis of Lyon (France) in a Climate Change Context. International Journal of Environmental Research and Public Health, 17(3),1004.

Bigot S., Duché S., Madelin M., Rome S., 2017. Etude du climat urbain : pour une mise à disposition de nouveaux services climatiques. BSGLg [En ligne], 68.

Cantat O., 2014. L'îlot de chaleur urbain parisien selon les types de temps. Norois [En ligne], 191.

Chapman L., Bell C., Bell S., 2017. Can the crowdsourcing data paradigm take atmospheric science to a new level? A case study of the urban heat island of London quantified using Netatmo weather stations. International Journal of Climatology, 37(9), 3597-3605.

de Vos L.W., Leijnse H., Overeem A., Uijlenhoet R., 2019. Quality Control for Crowdsourced Personal Weather Stations to Enable Operational Rainfall Monitoring. Geophysical Research Letters, 46(15), 8820-8829.

Dousset B., Gourmelon F., Laaidi K., Zeghnoun A., Giraudet E., Bretin P., Mauri E., Vandentorren S., 2011. Satellite monitoring of summer heat waves in the Paris metropolitan area. International Journal of Climatology, 31(2), 313-323.

Feichtinger M., de Wit R., Goldenits G., Kolejka T., Hollósi B., Žuvela-Aloise M., Feigl, J., 2020. Case-study of neighborhood-scale summertime urban air temperature for the City of Vienna using crowd-sourced data. Urban Climate, 32, 100597.

Fenner D., Holtmann A., Meier F., Langer I., Scherer D., 2019. Contrasting changes of urban heat island intensity during hot weather episodes. Environmental Research Letters, 14(12), 124013.

Fenner D., Meier F., Bechtel B., Otto M., Scherer D., 2017. Intra and inter local climate zone variability of air temperature as observed by crowdsourced citizen weather stations in Berlin, Germany. Meteorologische Zeitschrift, 26, 525-547.

Foissard X., 2015. L'îlot de chaleur urbain et le changement climatique : application à l'agglomération rennaise. Thèse de doctorat de Géographie, Université Rennes 2, 238 p. (avec annexes).

Foissard X., Dubreuil V., Quénol H., 2019. Defining scales of the land use effect to map the urban heat island in a mid-size European city: Rennes (France). Urban Climate, 29, 100490.

Hampel F. R., Ronchetti E. M., Rousseeuw P. J., Stahel W. A., 1986. Robust Statistics: The Approach Based on Influence Functions. Wiley, New York, 528 p.

Kastendeuch P., Najjar G., 2015. Une simulation des interactions ville-atmosphère à différentes échelles : application sur Strasbourg. Climatologie, 12, 44-64.

Kounkou-Arnaud R., Brion D., 2018. Optimal Interpolation of Daily Temperatures around Paris, taking into account Urban Fraction. International Conference of Urban Climate ICUC 10, New York, US.

Laaidi K., Zeghnoun A., Dousset B., Bretin P., Vandentorren S., Giraudet E. et al., 2012. The impact of heat islands on mortality in Paris during the August 2003 heat wave. Environmental health perspectives, 120, 2, 254-259.

Le Bras J., 2015. Le microclimat urbain à haute résolution : mesures et modélisation. Thèse de doctorat, Université Toulouse 3 Paul Sabatier, 208 p. (avec annexes).

Le Roy B., Lemonsu A., Kounkoud-Arnaud R., Brion D., Masson V., 2019. Long time series spatialized data for urban climatological studies: a case study of Paris, France. International Journal of Climatology, https://doi.org/10.1002/joc.6414.

Madelin M., Bigot S., Duché S., Rome S., 2017. Intensité et délimitation de l'îlot de chaleur nocturne de surface sur

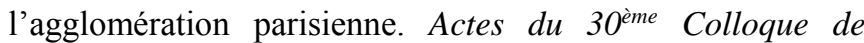
l'Association Internationale de Climatologie, Sfax, Tunisie, 39-45.

Madelin M., Dupuis V., 2018. Intensity and delimitation of the night urban heat island over the Paris metropolitan area from crowdsourced and remote sensing data. International Conference of Urban Climate ICUC 10, New York, US.

Mandement M., Caumont O., 2019. Contribution of Personal Weather Stations to the Observation of Deep-Convection Features Near the Ground. 18th Conference on Mesoscale Processes AMS.

Masson V., Gomes L., Pigeon G., Liousse C., Pont V., Lagouarde J.-P., Voogt J., Salmond J., Oke T. R., Hidalgo J., Legain D., Garrouste O., Lac C., Connan O., Briottet X., Lachérade S., Tulet P., 2008. The Canopy and Aerosol Particles Interactions in TOulouse Urban Layer (CAPITOUL) experiment. Meteorology and Atmospheric Physics, 102, 135 157.

Meier F., Fenner D., Grassmann T., Jänicke B., Otto M., Scherer, D., 2015. Challenges and benefits from crowd sourced atmospheric data for urban climate research using Berlin, Germany, as testbed. International Conference of Urban Climate ICUC 9, jointly with 12th Symposium on the Urban Environment.

Meier F., Fenner D., Grassmann T., Otto M., Scherer D., 2017. Crowdsourcing air temperature from citizen weather stations for urban climate research. Urban Climate, 19, 170-191.

Muller C. L., Chapman L., Grimmond C. S. B., Young, D. T., Cai, X., 2013. Sensors and the city: a review of urban meteorological networks. International Journal of Climatology, 33, 1585-1600.

Muller C. L., Chapman L., Johnston S., Kidd C., Illingworth S., Foody G., Overeem A., Leigh R. R., 2015. Crowdsourcing for climate and atmospheric sciences: current status and future potential. International J. of Climatology, 35, 11, 3185-3203. 
Nipen T. N., Seierstad I. A., Lussana C., Kristiansen J., Hov $\varnothing$., 2019. Adopting citizen observations in operational weather prediction. Bulletin of the American Meteorological Society, 101, E43-E57.

Oke T. R., 1982. The energetic basis of the urban heat island. Quarterly Journal of the Royal Meteorological Society, 108, 455, 1-24.

Oke T. R., 1987. Boundary Layer Climates. Routledge, 435 p.

Pascal M., Wagner V., Corso M., Laaidi K., Ung A., Beaudeau P., 2018. Heat and cold related-mortality in 18 French cities. Environment International, 121,189-198.

Richard Y., Emery J., Dudek J., Pergaud J., Chateau-Smith C., Zito S., Rega M., Vairet T., Castel T., Thévenin T., Pohl B., 2018. How relevant are local climate zones and urban climate zones for urban climate research? Dijon (France) as a case study. Urban climate, 26, 258-274.

Sakhy A., 2016. Îlots de chaleur et morphologie urbaine de l'agglomération parisienne : conséquences sur la mortalité durant la canicule de 2003. Thèse de doctorat en Géographie, Université Paris Diderot, 306 p. + annexes.

Skarbit N., Stewart I.D., Unger J., Gál, T., 2017. Employing an urban meteorological network to monitor air temperature conditions in the 'local climate zones' of Szeged, Hungary. International Journal of Climatology, 37, 582-596.

Stewart I. D., 2011. A systematic review and scientific critique of methodology in modern urban heat island literature. International Journal of Climatology, 31, 200-217.

Stewart I. D., Oke T. R., 2012. Local Climate Zones for Urban Temperature Studies. Bulletin of the American Meteorological Society, 93, 1879-1900.

Uteuov A., Kalyuzhnaya A., Boukhanovsky A., 2019. The cities weather forecasting by crowdsourced atmospheric data. Procedia Computer Science, 156, 347-356.

Venter Z. S., Brousse O., Esau I., Meier F., 2020. Hyperlocal mapping of urban air temperature using remote sensing and crowdsourced weather data. Remote Sensing of Environment, 242, 111791.

Wouters H., De Ridder K., Van Lipzig N., Demuzere M., Lauwaet D., 2013. The diurnal evolution of the urban heat island of Paris: a model-based case study during Summer 2006. Atmospheric Chemistry and Physics, 13(17), 85258541.

Citation de l'article : Madelin M. et Dupuis V., 2020. Intensité et spatialisation de l'îlot de chaleur urbain parisien à partir de données participatives. Climatologie, 17,9 . 